\title{
O silêncio da sexualidade em idosos dependentes
}

\author{
The silenced sexuality in dependent older adults
}

Konrad Gutterres Soares (https://orcid.org/0000-0002-5361-9419) ${ }^{1}$

Stela Nazareth Meneghel (https://orcid.org/0000-0002-7219-7178) ${ }^{1}$

${ }^{1}$ Programa de PósGraduação em Saúde Coletiva, Escola de Enfermagem, Universidade Federal do Rio Grande do Sul. R. São Manoel 963, Rio Branco. 90620-110. Porto Alegre RS Brasil.

konradgs@gmail.com

\begin{abstract}
Sexuality is a silent theme concerning the elderly population who is socially perceived as asexual. This qualitative approach paper is part of research on dependent older adults, which aimed to analyze their sexual experiences. The topic was retrieved from interviews with 26 of them, who talked about sex in their lives. The Thematic content analysis identified two main categories in the statements: gender differences in the treatment of sexuality and difficulty in talking about sex. Differences were observed in the perception and valuation of sexuality by gender. Older men give importance to sexual potency, and older women consider the cessation of sexual life in old age to be natural. Older men enunciate a sexuality concept based on biological satisfaction and sexual act, while women value partnership, affection, and fondness. Researchers were silent on the topic, although it was included in the research manual to guide the interview with older adults. However, while only 26 mentioned the subject, sexuality of older adults is modulated by gender perception. It is a poorly studied and challenging theme to approach in research and healthcare settings.
\end{abstract}

Key word Dependent older adults, Sexuality, Gender, Qualitative research
Resumo A sexualidade é um tema silenciado em relação à população idosa que é socialmente percebida como assexuada. Este artigo de abordagem qualitativa faz parte de uma pesquisa sobre idosos dependentes cujo objetivo foi analisar suas vivências em relação à sexualidade. O tema foi obtido a partir de entrevistas com 26 deles, que falaram sobre o sexo em suas vidas. A análise de conteúdo temática identificou duas categorias principais nas falas: diferenças de gênero no trato da sexualidade e dificuldade de falar sobre sexo. Observaram-se diferenças na percepção e valorização da sexualidade, segundo gênero. Homens idosos conferem importância para a potência sexual, $e$ mulheres idosas consideram natural a cessação da vida sexual na velhice. Os idosos do sexo masculino enunciam um conceito de sexualidade pautado na satisfação biológica e no ato sexual, enquanto as mulheres valorizam mais a parceria, o afeto $e$ o carinho. Constatou-se silêncio dos pesquisadores quanto ao tema, embora ele tenha sido colocado no manual de pesquisa para orientar a entrevista com a pessoa idosa. No entanto, embora apenas 26 tenham mencionado o assunto, foi possivel constatar que a sexualidade do idoso é modulada pela percepção de gênero e é um tema pouco estudado e de difícil abordagem em cenários de pesquisa e de atenção à saúde.

Palavras-chave Idosos dependentes, Sexualidade, Gênero, Pesquisa qualitativa 


\section{Introdução}

O declínio sexual como algo inevitável marca a reflexão sobre o envelhecimento como um preconceito, dentro do pressuposto de que os indivíduos deveriam se ajustar a esse imperativo, de modo a usufruir os benefícios morais da maturidade pós-sexual. No século XIX acreditava-se que um estilo de vida prudente retardaria esse declínio, mas aceitá-lo era parte do exercício moral de ajustamento aos efeitos do processo vital ${ }^{1}$.

Nos cenários atuais, porém, a busca pela manutenção da atividade sexual na velhice já é um consenso $^{2,3}$, embora mantenha-se no senso comum, inclusive de pesquisadores e profissionais de saúde, preconceitos e silêncios sobre esse assunto tabu, aumentando a vulnerabilidade dos idosos para problemas psicológicos e físicos, como doenças sexualmente transmissíveis e disfunção erétil ${ }^{4}$.

No processo de envelhecimento, mudanças significativas ocorrem com o corpo, incluindo a perda do desejo ou da potência sexual ${ }^{2}$, porém a maneira como a sexualidade é percebida e o significado que o envelhecimento assume são específicos de cada formação social e de cada momento histórico ${ }^{5,6}$.

A sexualidade pode ser definida, de maneira ampla, como a construção social dos usos do corpo e em particular, mas não exclusivamente, dos órgãos genitais, buscando obter prazer físico e mental. Compreende também a ordenação destas atividades, o que determina um conjunto de regras e normas que indicam, socialmente, as pessoas com as quais tais atividades podem ou não e devem ou não ser praticadas ${ }^{7}$.

Segundo a $\mathrm{OMS}^{8}$, a sexualidade é um componente central do ser humano, manifesta-se ao longo da vida e abrange sexo, identidades e papéis de gênero, orientação sexual, erotismo, prazer, intimidade e reprodução. Ela é experimentada e se expressa em pensamentos, fantasias, desejos, crenças, atitudes, valores, comportamentos, práticas, papéis e relacionamentos.

Fenômeno social complexo, a sexualidade é parte integrante da personalidade e seu desenvolvimento se completa enquanto necessidade humana básica como o desejo de contato, de intimidade, de expressão emocional, de prazer, de amor e de carinho?.

As concepções de gênero presentes na sociedade funcionam como normas e padrões que as pessoas devem seguir, incluído a atividade sexual. Nas sociedades patriarcais, as relações entre os sexos, marcadas pela dominação masculina ${ }^{10}$, determinam o que é considerado normal e interpretado como natural. Os padrões culturais em forma de estereótipos indicam que os homens, para demonstrar sua virilidade, precisam assegurar o direito à violência e à dominação das mulheres, bem como, afirmar sua potência sexual por meio do exercício de uma sexualidade erétil e penetrante ${ }^{11}$. Inseridos neste modelo, os homens idosos acreditam que devem ou precisam continuar exercendo a sexualidade enquanto prática do ato sexual e aqueles que, por alguma limitação não conseguem fazê-lo, sentem-se diminuídos e são acometidos de frustração e tristeza, o que pode acelerar o processo de envelhecimento e repercutir negativamente na saúde física e mental ${ }^{12}$.

As mulheres, por sua vez, são socializadas para satisfazer o desejo masculino, secundarizando os seus próprios, e podem, desta maneira, minimizar a importância do sexo e do prazer sexual. Atributos, como paciência, empatia e compaixão, são colocados como naturais e, desta maneira, elas ficam responsáveis socialmente pelo trabalho reprodutivo e de cuidado, atividades que operam no sentido de manter a hierarquia entre os sexos ${ }^{11}$.

O culto ao corpo jovem, idealizado pela sociedade atual, perpetua a convicção de que a sexualidade está ligada à juventude e à beleza ${ }^{13}$, o que redunda em preconceito em relação à sexualidade na velhice ${ }^{14}$, deserotizando o corpo e tratando-o como incapaz de produzir desejo ${ }^{15}$. Nesse sentido, as mulheres enfrentam maior desvantagem no campo simbólico, pois a construção social de suas identidades está fortemente associada à imagem corporal ${ }^{5}$.

Permanece na sociedade atual um preconceito que considera os idosos assexuados, o qual, aliado às dificuldades de falar sobre sexo, produz desconforto e constrangimento nas relações sociais e um silenciamento sobre este tema também entre pacientes e profissionais de saúde ${ }^{16}$. No campo da atenção à saúde, a temática da sexualidade do idoso tem sido negligenciada e a possibilidade de um idoso ser infectado pelo HIV, até pouco tempo, por exemplo, era considerada remota. Entretanto, dados nacionais referem que a taxa de HIV em idosos já supera a de adolescentes $^{17}$ e dentre as explicações para essas taxas elevadas estão o aumento das práticas sexuais na velhice $^{18}$.

Apesar de sua importância, a sexualidade do idoso é uma temática carente de pesquisas e a maioria dos estudos se detém nos aspectos fisiológicos negativos, como as disfunções sexuais ${ }^{19}$. Se internacionalmente são poucas as pesquisas, 
no Brasil elas são ainda mais escassas ${ }^{20}$, embora se tenha enfatizado, na pesquisa da qual este estudo faz parte, a necessidade de incluir esta questão ${ }^{21}$.

O objetivo deste artigo é analisar as vivências da sexualidade entre idosos e idosas com dependência, segundo o ponto de vista deles na pesquisa Estudo Situacional dos Idosos Dependentes que residem com suas Famílias visando a subsidiar uma Política de Atenção e de Apoio aos Cuidadores ${ }^{22}$.

\section{Método}

Neste artigo, o objetivo foi identificar as vivências relatadas por idosos dependentes sobre sua sexualidade após o estado de dependência ou durante a vida. Os dados foram produzidos por meio de entrevistas semiestruturadas, que incluíam uma conversa sobre sua sexualidade, dentre outros aspectos da vida no dia-a-dia. A questão era assim introduzida: "Conte, como é ou era o sexo para você”. Essa questão fez parte de um estudo qualitativo com idosos residentes em oito municípios brasileiros, visando a conhecer suas opiniões, crenças e representações a respeito do seu estado de dependência ${ }^{22}$.

Apenas 26 dos 64 idosos entrevistados falaram algo sobre sexualidade. Portanto, o estudo se concentra nas expressões desse pequeno grupo: moradores das cidades de Araranguá (3), Brasília (10), Manaus (3) e Porto Alegre (10).

Para compreender o que os idosos disseram sobre o sexo em suas vidas, utilizou-se a análise temática ${ }^{23,24}$, que considera as estruturas de relevância expressas nas falas, nas informações prestadas, nos argumentos e nas justificativas sobre o assunto.

Para melhor entender os cenários de vida destes idosos e as possibilidades de expressão da sexualidade foram produzidos pequenos relatos contextualizando a situação da doença e da dependência. Observou-se que o conteúdo da fala e a ênfase na enunciação variaram de acordo com o sexo dos entrevistados, indicando que a construção social de gênero - masculinidades e feminilidades - modula os comportamentos de homens e mulheres idosos em relação ao desempenho e às expectativas referentes à vida sexual. Considerou-se então que o gênero constitui uma categoria chave para a análise ou "gênero e sexualidade na vida de idosos e de idosas com dependência”.

A constatação de que o assunto não tenha sido tratado por boa parte dos pesquisadores que conduziram a conversa com os idosos representa em si, um indicador da dificuldade que sentem em falar sobre sexualidade. Esse fato gerou uma indagação sobre os motivos dessa dificuldade, o que acabou por dar origem a uma segunda categoria de análise explorada neste artigo, denominada "dificuldades de falar sobre sexo com idosos com dependência".

Essa pesquisa foi aprovada pela Comissão de Pesquisa da Escola de Enfermagem/UFRGS e pelo Comitê de Ética e Pesquisa da Universidade Federal do Rio Grande do Sul.

\section{Gênero e sexualidade na vida de idosos e de idosas com dependência}

Neste artigo trabalhou-se com uma amostra de 12 homens e 14 mulheres idosos com dependência. Desses, 17 se consideraram brancos, oito pardos ou pretos e um não informou sobre cor. Sete deles havia cursado mais de 11 anos de estudo e mais da metade professava a religião católica. A maioria, 16 idosos, vivia com companheiro (a) e relatou manter atividades de convívio social.

Nas entrevistas realizadas com os idosos do sexo masculino, observou-se que eles expressam uma valorização do sexo e da sexualidade relacionada aos aspectos biológicos, portanto, à capacidade de realizar o ato sexual e à presença ou não de penetração. Para eles, sexo significa relação sexual, relação sexual significa penetração vaginal e penetração vaginal requer ereção peniana.

O impasse vivido por alguns deles em situações em que há desejo sexual, ao mesmo tempo em que ocorre perda da função sexual, provoca sentimentos de angústia, decepção, frustração e medo de fracassar; e em alguns gera tristeza, sofrimento e depressão. Esse quadro ratifica a percepção de que a disfunção erétil atinge profundamente o coração da masculinidade, fazendo que os homens sintam que perderam a vitalidade, aquilo que os faz homens ${ }^{25}$.

Não sei se a mulher tem essa dificuldade, o homem, como machista, é pior ainda e já vai para quatro anos isso. É cruel guri! É cruel, é a pior parte de todas (Idoso, 65 anos, Porto Alegre).

Essa fala foi enunciada por um homem relativamente jovem, que possui uma doença neurológica degenerativa. Antes da doença, era o provedor da família, centrava sua vida no trabalho e nos ganhos financeiros. No início da entrevista, ele dizia que o que mais o afetava era não poder trabalhar. Porém, quando perguntado sobre o exercício da sexualidade, afirmou que não se tratava do trabalho, o sexo era o que mais importava na sua vida, colocando a perda da potência sexual 
como a pior consequência da doença. A disfunção sexual desse idoso e a perda do trabalho o levaram a se sentir inútil e deprimido, um inválido. Na concepção cultural reducionista da vivência da sexualidade somente por meio do coito, alguns idosos sentem-se diminuídos, pois o ato sexual é afetado por alterações corporais inexoráveis provenientes da idade, tais como, disfunção erétil e alterações hormonais ${ }^{26}$.

A vida da gente terminou. Terminou-se! (Idoso, 72 anos, Araranguá).

Acabou tudo. Eu vivia com a minha mulher muito bem, mas vai chegando a velhice... (Idoso, 88 anos, Manaus).

Os depoimentos destes dois idosos, moradores respectivamente do sul e norte do país, trazem a declaração enfática do término da vida sexual, mostrando sentimentos de perda e irreversibilidade. $O$ primeiro deles sofreu um acidente vascular e possui pouca autonomia. A enfermidade lhe trouxe a disfunção erétil, além de perdas funcionais que interferiram em seus planos de futuro. $O$ segundo possui um histórico de várias morbidades. Relata que ele e a companheira não praticam mais sexo e, inclusive, dormem em camas separadas. Associa o distanciamento na relação com a esposa com a perda da potência sexual. Sente-se triste, isolado e, como a maioria dos idosos entrevistados, inválido. Ambos consideram a sexualidade como sinônimo de ato sexual e não cogitam outras formas de expressão, quer seja verbal ou por meio de carinhos, toques e outras manifestações de desejo e afeto. De certa maneira, os depoimentos corroboram o fato de que a presença de enfermidade está relacionada com a ausência ou a diminuição da prática sexual, principalmente quando a doença ocorre no homem e afeta a potência masculina ${ }^{27}$.

Com o estereótipo da assexualidade dos idosos, pode-se dizer que a sociedade contribui para que o idoso tenha a percepção de menos valia, porque as pessoas de mais idade sempre foram imaginadas como aquelas que estão se despedindo da vida. Deduz-se então que, por ter se aposentado do trabalho, o idoso se aposentou da vida, inclusive a sexual.

Agora está normal! Agora eu até nem lembro mais, mas é brabo. No início, foi brabo, mas agora está normalizado. Eu já aceito tudo mais (Idoso, 75 anos, Porto Alegre).

Bah, agora até já aceitei um pouco, mas, no início, era preocupante. Eu ficava atacado. Era uma barbaridade (Idoso, 65 anos, Porto Alegre).

Alguns idosos demonstram aceitação das perdas funcionais, como apontam as falas que se referem "à normalidade" de não haver mais sexo na relação conjugal. Esses dois idosos possuem histórico de doenças crônicas e ambos não partilham a cama com suas esposas. Um deles permanece a maior parte do tempo sentado numa poltrona da sala, onde costuma dormir. Ambos relataram que há muitos anos não mantêm relações sexuais. Frente à visão reducionista da sexualidade como a prática do ato sexual, o afastamento físico, no caso dormir em camas separadas ou em outro aposento, parece ter sido um arranjo para viverem o luto do término da vida sexual.

Poxa, coisa boa (risada)! Coisa boa que Deus deu para a gente! Eu digo assim, se não tivesse a gulosidade e o prazer não teria o mundo, não é? Não teria o mundo porque a gulosidade e o prazer são o que deixam o mundo em pé! Mas vamos levando. Fazer o quê? (Idoso, 82 anos, Porto Alegre).

Este homem de 82 anos parece lidar com a questão de maneira mais aberta e, apesar das limitações que a idade trouxe, ainda se mantém sexualmente ativo. Relata uma internação hospitalar recente, complicações e perda de autonomia, mas com ganho progressivo de funcionalidades. Frequenta bailes da terceira idade, possui uma boa relação com a esposa, dormem na mesma cama, mantêm relações sexuais. Mostra que está envelhecendo de maneira saudável, mesmo com dependência, mantendo tanto a vida sexual quanto o bom humor.

Esse e outros idosos, ao contrário da maioria, mostram que a sexualidade continua na velhice, vivida e usufruída por toda a vida ${ }^{4}$. Embora o processo de envelhecimento passe por mudanças físicas, bioquímicas e funcionais que interferem na atividade sexual, eles mantêm interesse sexual aos 70, 80 e mesmo 90 anos de idade ${ }^{28}$, interesse manifesto por meio de carinhos, toques e companheirismo ${ }^{29}$.

As idosas expressaram uma visão distinta da sexualidade, embora da mesma maneira que os homens, marcada pela cultura e pelas convenções sociais. Essas mulheres foram educadas meio século atrás, em um momento em que os padrões de gênero eram mais rígidos, ditando o que seria próprio ou impróprio para cada um dos sexos. As condutas em relação à sexualidade estavam impregnadas de normas, preconceitos e moralismos, e era mais comum a submissão e a subordinação ao marido ${ }^{30}$. Nas entrevistas, observam-se os diversos sentidos atribuídos pelas idosas que falaram sobre a sexualidade, ressaltando histórias de vidas marcadas pela repressão de desejos e afetos ${ }^{31}$.

Foi normal, porque eu não fiz mais com ninguém e eu não sinto falta! (Idosa, 78 anos, Porto Alegre). 
Eu não estou sentindo vontade. Não me importo de não sentir, porque não tenho companheiro (Idosa, 72 anos, Porto Alegre).

É importante, mas não sei se ele foi tão importante na minha vida, porque depois dele não sent $i$ mais falta (Idosa, 74 anos, Porto Alegre).

Os trechos selecionados foram enunciados por idosas viúvas, que após a perda do companheiro não se permitiram outras experiências e afirmam que a sexualidade nunca foi algo relevante em suas vidas e que não sentem falta de sexo. Mesmo as que assumem ainda sentirem desejo sexual não se permitem outras relações. Consideram que por não possuírem marido, isso não seria adequado.

A socialização de gênero em sociedades conservadoras supõe que mulher que não tem homem não pode ou não deve fazer sexo e muito menos ter prazer. No caso das idosas, as transformações corporais e a mensagem de uma cultura que valoriza a juventude e invisibiliza o velho, sustentam a impossibilidade de serem vistas como atraentes e desejáveis ${ }^{30}$. Os aspectos socioculturais reforçam os padrões de gênero, pois o que se espera da mulher é submissão, dependência e cuidado com o outro. É o que se observa nos depoimentos em que elas incorporam condutas que incluem a abdicação da sexualidade junto com a de si mesmas ${ }^{32}$.

Quando a gente fica velho, a gente perde tudo! (Idosa, 79 anos, Manaus).

O problema é ficar sem nada, sabe? Ficar assim inútil. Eu me considero uma pessoa inútil, sem utilidade nenhuma. Fico aqui parada "sem eira nem beira" (sem perspectiva) (Idosa, 67 anos, Porto Alegre).

As perdas funcionais e as alterações que o tempo impõe aos corpos ceifam a potência do masculino e, de modo diverso, mas tão duro quanto, significam perdas à potência do feminino. A declaração da moradora de Manaus de 79 anos e portadora de hanseníase, diz das perdas devidas à idade, mas também das alterações estéticas graves produzidas pela patologia. A imagem corporal tem significado e importância na prática do sexo, principalmente para as mulheres, de modo que a insatisfação com a aparência deixa muitas delas inseguras, levando-as a negar ou a bloquear a possibilidade de encetar relações afetivas. De tal modo que a não aceitação de si significa por decorrência, recusa ao sexo ${ }^{27,33}$.

O importante é tu ter uma pessoa para conversar, nem que seja pra brigar! Isso é importante! Sentar, conversar, para mim isso é que é importante (Idosa, 63 anos, Porto Alegre).
Fiquei quinze anos sem dormir com ele, mas nós éramos os melhores amigos que tínhamos (Idosa, 76 anos, Araranguá).

Eu sinto falta da companhia dele! De outro de jeito, não! (Idosa, 83 anos, Manaus).

A sexualidade é comumente considerada uma função fisiológica, minimizando os aspectos emocionais e subjetivos, os sentimentos e os afetos, o companheirismo e a ternura entre os pares $^{29}$. Porém, algumas das mulheres acenaram com essa dimensão mais ampla, focando vivências que não incluem aspectos físicos apenas, e trazem à tona uma visão da interação sexual como um processo complexo em que amizade, empatia, capacidade de comunicação e mesmo espiritualidade fazem parte ${ }^{19}$.

Eu tinha sexualidade para dar e vender! Há pouco tempo, fui fazer exame ginecológico e enfermeira me perguntou sobre sexo. Eu disse: sou velha, mas eu namoro igual a todo mundo! (Idosa, 84 anos, Araranguá).

Essa mulher de 84 anos e cadeirante, oriunda de família conservadora e que havia sido casada com um homem também cadeirante, após ficar viúva redescobriu sua sexualidade. O depoimento mostra uma abertura em relação às sempre presentes possibilidades de refazer e recriar as relações amorosas, reiterando o fato de que a sexualidade permeia todas as fases do ciclo vital humano, do nascimento à morte ${ }^{6}$.

Enfim, em relação ao gênero, os idosos do sexo masculino manifestaram uma percepção de sexualidade ligada ao biológico e à realização do ato sexual, mostrando intenso sofrimento frente às limitações fisiológicas com que se defrontam por causa do adoecimento. As mulheres, por sua vez, afirmam não sentir necessidade de sexo, treinadas que foram para controlar ou esconder seus próprios desejos. Mas há idosos que desfrutam de relações prazenteiras mesmo em idade avançada e idosas que percebem o exercício da sexualidade além do biológico, incluindo afeto, ternura e companheirismo.

Masculinidades e feminilidades saudáveis significam a capacidade de habitar e amar o próprio corpo, desfrutar dele nas relações eróticas e aceitar perdas e limitações ${ }^{11}$. As situações de envelhecimento e doença, em que a obrigatoriedade masculina de demonstrar potência e virilidade a qualquer custo e conformismo feminino de encerrar a vida sexual sem divisar possibilidades de ultrapassar as perdas, podem se tornar dolorosas e insuportáveis.

Neste estudo, fica clara a variabilidade modulada pelo gênero ${ }^{34}$ nas percepções e vivências 
relatadas pelos entrevistados ao falarem sobre o sexo em suas vidas, deixando evidente o quanto os matizes de gênero moldam comportamentos e expectativas distintas em relação ao exercício da sexualidade na velhice.

\section{Dificuldades de falar sobre sexo com idosos com dependência}

Questões sobre a sexualidade do idoso dependente, que faziam parte do roteiro da pesquisa, não foram investigadas e questionadas em mais da metade das entrevistas. Os entrevistadores, todos profissionais de saúde ou estudantes do campo, apresentaram dois tipos de justificativa por não terem formulado a questão: $o$ idoso não demonstrou interesse no assunto ou o entrevistador sentiu-se desconfortável em perguntar.

Ao afirmarem o desconforto para conversar sobre sexualidade, pode-se pensar na dificuldade do pesquisador como uma projeção cultural do preconceito de que esse assunto não seja algo adequado ao idoso. Na realidade, as pesquisas $\mathrm{s}^{35}$ sugerem que tanto na área acadêmica quanto na atenção à saúde, os profissionais têm pouco conhecimento sobre a sexualidade no envelhecimento e não conseguem lidar adequadamente com o tema. Em consequência, demonstram atitudes negativas ou evitam falar no assunto.

No caso dos profissionais de saúde, há pelo menos dois problemas na abordagem da temática. Por um lado, sentem-se inibidos em fazer perguntas de cunho sexual, considerando-as como falta de respeito. De outro, é comum o idoso ficar envergonhado e não ter coragem de fazer perguntas sobre o assunto, porque teme ser mal interpretado. Por causa do desconhecimento, do tabu e da pressão cultural, muitos experimentam sentimentos de culpa e de vergonha por sentirem desejo sexual ${ }^{36}$. Essas podem ser as mesmas razões que afetaram os pesquisadores, em sua maioria, pertencentes ao campo da saúde.

As barreiras que desencorajam a discussão sobre sexualidade com pessoas mais velhas são múltiplas: falta de confiança e de experiência, pouco conhecimento, vergonha e preconceitos em relação ao tema ${ }^{37}$. De acordo com Pascual ${ }^{38}$, existe, em nossa sociedade, um conceito de velhice negativo, especialmente no âmbito sexual, desta maneira não se fala sobre o assunto, os idosos são considerados assexuados, e os familiares colocam obstáculos para impedir que eles continuem sendo sexualmente ativos.

Profissionais de saúde e pesquisadores de campo são membros das sociedades onde estão inseridos, desta maneira podem compartilhar com entrevistados e com a população, pontos de vista moralistas ou conservadores e manifestar opiniões que refletem apenas noções do senso comum $^{39}$.

Na pesquisa sobre idosos dependentes, o elevado número de idosos para quem a pergunta sobre sexualidade não foi formulada, demonstra constrangimento do entrevistador ou da entrevistadora. $\mathrm{O}$ silêncio sinaliza mal estar frente a esse tema delicado, particularmente frente ao idoso em estado de limitação e dependência.

O silêncio pode também chamar atenção para a necessidade de falar sobre um assunto interditado com o maior número possível de atores, idosos, familiares, cuidadores, profissionais da saúde, pesquisadores e gestores, contribuindo para desmistificar preconceitos e acenar para a ideia de que não há idade limite para encerrar a atividade sexual.

\section{Considerações finais}

O envelhecimento ativo preconiza a possibilidade de se praticar sexo até o fim da vida ${ }^{2,19}$, nesse sentido, a inclusão dessa questão em uma pesquisa sobre idosos com dependências constituiu uma oportunidade de saber, no limite, como os idosos e idosas vivenciam as dificuldades nesta área.

Uma das limitações deste artigo é trabalhar com uma amostra relativamente pequena de participantes que falaram sobre o tema. No entanto, o que se ouviu demonstra o quanto a questão sexual é relevante, seja pelos tabus que carrega seja pela repressão que implica em sofrimento para algumas pessoas idosas, que continuam a ter desejos e querem se relacionar. Nesse sentido, a pesquisa contribuiu para a desconstrução da noção de que os idosos são assexuados ${ }^{40}$, entendendo que exercício da sexualidade faz parte desta etapa da vida ${ }^{41,42}$.

Acredita-se que conversar abertamente sobre sexo com idosos, inclusive com os que apresentam dependência e, portanto, possuem limitações para o seu exercício, contribui para romper os preconceitos e os tabus que cercam esse assunto. 


\section{Colaboradores}

KG Soares: Trabalho de campo, condução das entrevistas, participação em reuniões de discussão sobre dados coletados em Porto Alegre e na Fiocruz no Rio de Janeiro, revisão bibliográfica, elaboração do artigo, revisão do Artigo. SN Meneghel: Participação em reuniões de discussão sobre dados coletados em Porto Alegre e na Fiocruz no Rio de Janeiro, revisão bibliográfica, elaboração do artigo, revisão do artigo.

\section{Referências}

1. Karsch UM. Idosos dependentes: famílias e cuidadores. Cad Saude Publica 2003; 19(3):861-866.

2. Debert G, Brigeiro M. Fronteiras de gênero e a sexualidade na velhice. Rev Bras. Ci Soc 2012; 27(80):37-54.

3. Cezar AK, Aires M, Paz AA. Prevention of sexually transmitted diseases in the point of view of elderly clients of a Family Health Strategy. Rev Bras Enferm 2012; 65(5):745-750.

4. Frugoli A, Magalhães Júnior CAO. Sexuality in third age in the perception of a female elderly group and indications for the enviromental education. Arq Ciênc Saúde UNIPAR 2011; 15(1):85-95.

5. Silva VXL, Marques APO, Fonseca JLCL. Considerações sobre a sexualidade dos idosos nos textos gerontológicos. Rev Bras Geriatr Gerontol 2009; 12(2):295303.

6. Almeida T, Lourenço ML. Envelhecimento, amor e sexualidade: utopia ou realidade? Rev Bras de Geriatr Gerontol 2007; 10(1):101-113.

7. Lhomond B. Sexualidade. In: Hirata H, Laborie F, Le Doaré H, Senotier D, organizadores. Dicionário Crítico do feminismo. São Paulo: Ed. Unesp; 2009. p. 231235.

8. World Health Organization (WHO). Sexual Health, Human Rights and the Law. Geneva: WHO; 2015.

9. Moraes KM, Vasconcelos DP, Silva ASR, Silva RCC, Santiago LMM, Freitas CASL. The beauty of companionship and sexuality for couples in the best age: caring for elderly couple. Rev Bras Geriatr Gerontol 2011; 68(4):662-667.

10. Scott J. Gênero: uma categoria útil de análise histórica. Educação e Realidade 1995; 20(2):71-99.

11. Molinier P, Welzer-Lang D. Feminilidade, masculinidade, virilidade. In: Hirata H, Laborie F, Le Doaré H, Senotier D, organizadores. Dicionário Crítico do feminismo. São Paulo: Ed. Unesp; 2009. p. 101-104.

12. Uchoa YS, Costa DCA, Silva Jr IAP, Silva STSE, Freitas WMTM, Soares SCS. A sexualidade sob o olhar da pessoa idosa. Rev Bras Geriatr Gerontol 2016; 19 (6):939-949.

13. Goldenberg M. Mulheres e envelhecimento na cultura brasileira. Caderno Espaço Feminino 2012; 25(2):4656.

14. Linhares FMP, Pottes AF, Araújo EC, Menezes EP, Siqueira KA. Percepção de idosos sobre o exercício da sexualidade atendidos no Núcleo de Atenção ao idoso em Recife, Brasil. Rev Enferm Herediana 2008; 1(2):93-103.

15. Coelho DNP, Daher DV, Santana RF, Espírito Santo FH. Percepção de mulheres idosas sobre sexualidade: implicações de gênero e no cuidado de enfermagem. Revista Rene 2010; 11(4):163-173.

16. Gewirtz-Meydan A, Hafford-Letchfield T, Benyamini Y, Phelan A, Jackson J, Ayalon L. Ageism and sexuality. In: Ayalon L, Tesch-Römer C, editors. Contemporary perspectives on ageism. International Perspectives on Aging. Cham: Springer International Publishing AG; 2018. p.149-162.

17. Santos AFM, Assis M. Vulnerabilidade das idosas ao HIV/AIDS: despertar das políticas públicas e profissionais de saúde no contexto da atenção integral: revisão de literatura. Revista Brasileira de Geriatria e Gerontologia 2011; 14(1):147-157. 
18. Silva J, Saldanha AAW. Vulnerabilidade e convivência com o HIV/AIDS em pessoas acima de 50 anos. Revista Mal Estar e Subjetividade 2012; 12(3-4):817-852.

19. Vieira KFL, Coutinho MPL, Saraiva ERA. A sexualidade na velhice: representações sociais de idosos frequentadores de um grupo de convivência. Psicologia: Ciência e Profissão 2015; 36(1):196-209.

20. Moreira Júnior ED, Glasser D, Santos DB, Gingell C. Prevalence of sexual problems and related help-seeking behaviors among mature adults in Brazil: data from the global study of sexual attitudes and behaviors. Sao Paulo Med J 2005; 123(5):234-241.

21. Brasil. Ministério da Saúde (MS). Envelhecimento saúde da pessoa idosa. Saúde da Família. Brasília: MS; 2006. (Cadernos de Atenção Básica 19).

22. Minayo MCS, Figueiredo AEB, Mangas RMN, Silva RM, Vieira LJES, Souza GS, Meneghel SN, Gutierrez DMD, Figueiredo MLF, Mendonça JMB. Estudo Situacional dos Idosos Dependentes. Rio de Janeiro: ClavesFiocruz; 2019.

23. Minayo MCS. O Desafio do Conhecimento. Pesquisa Qualitativa em Saúde. 14a. ed. Rio de Janeiro: Hucitec; 2012.

24. Bardin L. Análise de conteúdo. Lisboa: Edições 70; 1977.

25. Gewirtz-Meydan A, Hafford-Letchfield T, Ayalon L, Benyamini Y, Biermann V, Coffey A, Jackson J, Phelan A, Voß P, Zeman MG, Zeman Z. How do older people discuss their own sexuality? A systematic review of qualitative research studies. Cult Health Sex 2019; 21(3):293-308.

26. Queiroz MAC, Lourenço RME, Coelho MMF, Miranda KCL, Barbosa RGB, Bezerra STF. Representações sociais da sexualidade entre idosos. Rev Bras Enferm 2015, 68(4):662-667.

27. Alencar DLM, Marques APO, Leal MCC, Vieira JCM. Fatores que interferem na sexualidade de idosos: uma revisão integrativa. Cien Saude Colet 2014; 19(8):3533-3542.

28. Gott M, Hinchliff S. How important is sex in later life? The views of older people. Soc Sci Med 2003; 56(8):1617-1628.

29. Bastos CC, Closs VE, Pereira AMVB, Batista C, Idalêncio FA, Carli GA, Gomes I, Schneider RH. Importância atribuída ao sexo por idosos do município de Porto Alegre e associação com a autopercepção de saúde e o sentimento de felicidade. Rev Bras Geriatr Gerontol 2012; 15(1):87-95.

30. Oliveira EL, Neves ALM, Silva IR. Sentidos de Sexualidade Entre Mulheres Idosas: Relações de Gênero, Ideologias, Mecanicistas e Subversão. Psicol Soc 2018; 30:e166019.

31. Botacci LFG. A construção social do sexo: alguns aspectos a considerar sobre a terceira idade. Revista Trilhas da História 2011; 1(1):145-158.
32. Souza M, Marcon SS, Bueno SMV, Carreira L, Baldissera VDA. A vivência da sexualidade por idosas viúvas e suas percepções quanto à opinião dos familiares a respeito. Saude Soc 2015; 24(3):936-944.

33. Alencar DL, Marques APO, Leal MCC, Vieira JCM. O exercício da sexualidade em pessoas idosas e os fatores relacionados. Rev Bras Geriatr Gerontol 2016; 19(5):861-869.

34. Jen S. Older women and sexuality: Narratives of gender, age, and living environment, J Women Aging 2016; 29(1):1-11.

35. Bauer M, Haesler E, Fetherstonhaugh D. Let's talk about sex: older people's views on the recognition of sexuality and sexual health in the health-care setting. Health Expect 2016; 19(6):1237-1250.

36. Vieira KLF. Sexualidade e qualidade de vida do Idoso: desafios contemporâneos e repercussões sociais [tese]. João Pessoa: Universidade Federal da Paraíba; 2012.

37. Heath H. Sexuality and sexual intimacy in later life. Nursing Older People 2019; 31(1):40-48.

38. Pascual CP. A sexualidade do idoso vista com novo olhar. São Paulo: Loyola; 2002.

39. Meneghel SN, Andrade DNP. Conversas entre mulheres durante o exame citopatológico. Saude Soc 2019; 28(2):174-186.

40. Fileborn B, Lyons A, Hinchliff S, Brown G, Heywood W, Dow B, Malta S, Minichiello V. Improving the sexual lives of older Australians: Perspectives from a qualitative study. Australas J Ageing 2017; 36(4):E3642.

41. Gewirtz-Meydan A, Levkovich I, Mock M, Gur U, Karkabi K, Ayalan L. Sex for seniors: how physicians discuss older adult's sexuality. Isr J Health Policy Res 2020; 9(1):8

42. Evangelista AR, Moreira ACA, Freitas CASL, Val DR, Diniz JL. Sexualidade de idosos: conhecimento/atitude de enfermeiros da Estratégia Saúde da Família. Rev Esc Enferm USP 2019; 53:e03482.

Artigo apresentado em 14/07/2020

Aprovado em 13/08/2020

Versão final apresentada em 15/08/2020

Editores chefes: Maria Cecília de Souza Minayo, Romeu Gomes, Antônio Augusto Moura da Silva. Editora Associada da área de Saúde do Idoso: Joselia Oliveira Araújo Firmo 